\title{
Spatially controlled epitaxial growth of 2D heterostructures via defect engineering using a focused $\mathrm{He}$ ion beam
}

\author{
Martin Heilmann ${ }^{1,4 凶}$, Victor Deinhart ${ }^{2,3}$, Abbes Tahraoui ${ }^{1}$, Katja Höflich ${ }^{3,5}$ and J. Marcelo J. Lopes ${ }^{1 凶}$
}

The combination of two-dimensional (2D) materials into heterostructures enables the formation of atomically thin devices with designed properties. To achieve a high-density, bottom-up integration, the growth of these 2D heterostructures via van der Waals epitaxy (vdWE) is an attractive alternative to the currently mostly employed mechanical transfer, which is problematic in terms of scaling and reproducibility. Controlling the location of the nuclei formation remains a key challenge in vdWE. Here, a focused He ion beam is used to deterministically place defects in graphene substrates, which serve as preferential nucleation sites for the growth of insulating, 2D hexagonal boron nitride (h-BN). Therewith a mask-free, selective-area vdWE (SAvdWE) is demonstrated, in which nucleation yield and crystal quality of h-BN are controlled by the ion beam parameters used for defect formation. Moreover, $\mathrm{h}$-BN grown via SAvdWE is shown to exhibit electron tunneling characteristics comparable to those of mechanically transferred layers, thereby lying the foundation for a reliable, high-density array fabrication of $2 \mathrm{D}$ heterostructures for device integration via defect engineering in 2D substrates.

npj 2D Materials and Applications (2021)5:70; https://doi.org/10.1038/s41699-021-00250-z

\section{INTRODUCTION}

Through the combination of two-dimensional (2D) materials, novel van der Waals heterostructures with tailored properties can be designed for applications in atomically thin and transparent optoelectronic, electronic, and sensing devices ${ }^{1-4}$. However, the currently most employed integration via mechanical transfer remains challenging with regard to reliability as well as scalability for a future ultrasmall-size device fabrication. Therefore, the growth of 2D materials on top of each other via van der Waals epitaxy (vdWE) is an attractive alternative to form atomically thin heterostructures ${ }^{1,5,6}$.

Within 2D heterostructures, hexagonal boron nitride (h-BN) plays a central role in two ways: Electronically, its large band-gap enables efficient electronic confinement (as required in ultra-thin layers) ${ }^{3}$, while its chemical inertness (i.e. the lack of dangling bonds), together with its insulating nature and high mechanical strength, make it an ideal substrate or encapsulation layer for other 2D materials ${ }^{7}$. A detailed overview of various techniques used for the synthesis of h-BN can be found elsewhere ${ }^{8}$. Scalable fabrication techniques such as chemical vapor deposition or molecular beam epitaxy (MBE) were used for the growth of h-BN/ graphene heterostructures $^{9-12}$. The weak out-of-plane interactions between 2D materials lead to preferential nucleation at defects and morphological features in the $2 \mathrm{D}$ substrates in vdWE, giving rise to non-uniform growth ${ }^{12-15}$. Up to now there is no feasible way to control the exact location and orientation for the nucleation of 2D materials, making it one of the key challenges to achieve high-quality material for electronic applications ${ }^{16}$. Previous studies on the selective heteroepitaxy on vdW substrates concentrated on the growth of multiple $2 \mathrm{D}$ layers on mica or $3 \mathrm{D}$ nanostructures on graphene using photo- or electron beam lithography and plasma etching for processing a mask ${ }^{17-20}$. However, these processes have a limited resolution (particularly in the case of photolithography) and the masking process threatens to contaminate or damage the 2D substrates.

Instead of a mask, we used a focused ion beam (FIB) within a $\mathrm{He}$ ion microscope to deliberately create atomic-scale defects in epitaxial graphene (EG) on $\mathrm{SiC}$ with perfect position control. The use of EG as a substrate is advantageous for two reasons: (i) the sublimation of $\mathrm{Si}$ from $\mathrm{SiC}$ leads to a reproducible formation of epitaxially aligned graphene with low intrinsic defect density and (ii) it can be directly synthesized on semi-insulating $\mathrm{SiC}$ with no need for a transfer process. According to molecular dynamics simulations, irradiation of 2D materials with ions of noble gases with energies in the keV range results in the formation of defects with control down to single vacancies ${ }^{21-23}$. In this regard, a He FIB was recently used to write optical active defects in $\mathrm{MoS}_{2}{ }^{24}$. In our approach, the irradiation-induced defects (IIDs) in EG exhibit dangling bonds, which act as preferential nucleation sites for atomically thin $\mathrm{h}-\mathrm{BN}$ grown from elemental $\mathrm{B}$ and $\mathrm{N}$ sources via plasma-assisted MBE (PAMBE) (Fig. 1a-c). Our mask-free SAvdWE of $h-B N$ is superior to photo- or electron beam lithography, as no residues from the mask threatens to contaminate the surface of the 2D substrate. Ideally, only a single $C$ atom needs to be removed from pristine graphene, as graphene itself serves as a mask through its chemically inert nature. Unlike heavier Ga ions, which have been used for the formation of nanoholes in graphite flakes and the selective growth of few-layer h-BN within, the lighter $\mathrm{He}$ ions are better suitable to form atomic-scale defects in $2 \mathrm{D}$ substrates and therefore for the selective growth of atomically thin heterostructures ${ }^{21,25}$.

\section{RESULTS AND DISCUSSION}

Deterministic placement of defects in graphene via He FIB

The processing of EG on semi-insulating $\mathrm{SiC}$ substrates results in step edges on the surface with up to $20 \mathrm{~nm}$ height (visible in the

\footnotetext{
${ }^{1}$ Paul-Drude-Institut für Festkörperelektronik, Leibniz-Institut im Forschungsverbund Berlin e.V., Berlin, Germany. ${ }^{2}$ Ferdinand-Braun Institut gGmbH, Leibniz-Institut für Höchstfrequenztechnik, Berlin, Germany. ${ }^{3}$ Helmholtz-Zentrum Berlin für Materialien und Energie GmbH, Berlin, Germany. ${ }^{4}$ Present address: IMEC, Leuven, Belgium. ${ }^{5}$ Present address: Ferdinand-Braun Institut gGmbH, Leibniz-Institut für Höchstfrequenztechnik, Berlin, Germany. ${ }^{凶}$ email: martinheilmann@gmx.de; lopes@pdi-berlin.de
} 

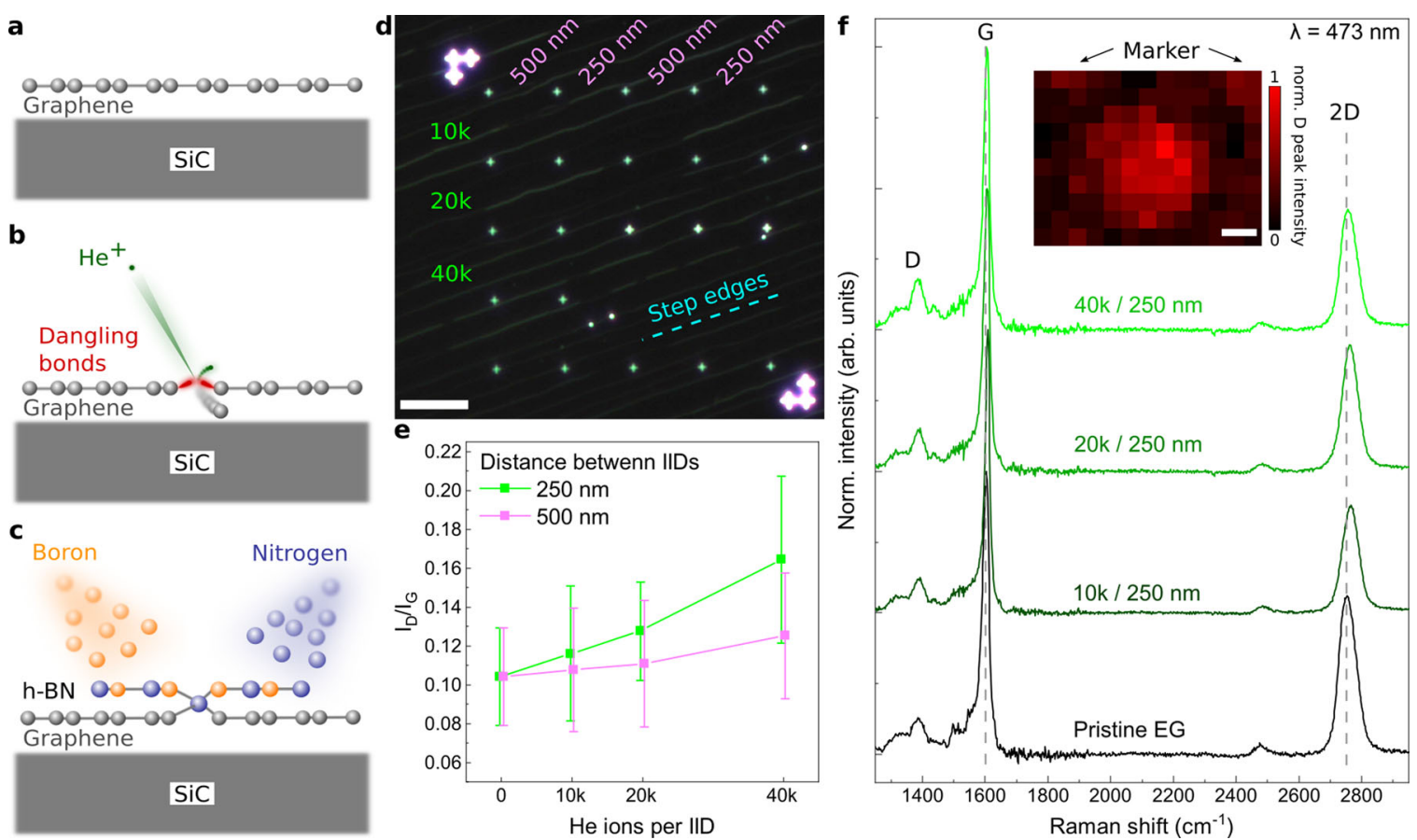

Fig. 1 Defect-engineering in EG on SiC via He FIB. a-c Schematic representation of the SAvdWE process. a Pristine EG fabricated via Si sublimation from SiC substrates. b Deterministic placement of IIDs in EG via He FIB. c Growth of h-BN via PAMBE, where dangling bonds at the defects act as preferential nucleation sites. For simplicity the C-rich interface layer to the SiC was neglected in these images. d Darkfield micrograph from structured area showing the $4 \times 3$ matrix of the FIB pattern, each containing $19 \times 19$ IIDs, written in a square grid with varying distance to each other and different number of $\mathrm{He}$ ions as indicated by magenta and green annotations, respectively. SiC step edges run parallel to the cyan dotted line. Additional cross-shaped markers were written between the different areas via He FIB. e Average ratio between D and G Raman peak intensity for 36 spectra recorded in each area in the FIB pattern. The two different data sets are laterally offset for clarity. The error bars represent one standard deviation. $f$ Comparison of the mean Raman spectra of patterns with higher IID density written with varying number of He ions per defect. The spectra were normalized with respect to the $\mathrm{G}$ peak and vertically shifted for clarity. The gray dotted lines mark the position of the $G$ and $2 D$ peaks in pristine EG. Inset: Raman map of the $D$ peak intensity marking the structured area containing $19 \times 19 \mathrm{IIDs}$, each written with $40,000 \mathrm{He}$ ions in a distance of $250 \mathrm{~nm}$ to each other, in between markers (indicated by black arrows). Scale bars, $10 \mu \mathrm{m}$ in (d), $2 \mu \mathrm{m}$ in the inset of (f).

dark-filed micrograph in Fig. 1d) and several $\mu \mathrm{m}$ wide terraces in between (see Supplementary Fig. 1), which are covered with single- (SLG) and bi-layer graphene (BLG). Using a He FIB with an energy of $30 \mathrm{keV}$ and focal spot sizes below $1 \mathrm{~nm}$, the IIDs were written in a pattern, which was aligned to marker structures on the $\mathrm{SiC}$ substrate (see method section as well as Supplementary Fig. 1). The He FIB patterns are located in between three cross-shaped markers in the upper left and lower right corners, encompassing a structured area of $40 \times 40 \mu^{2}$, as visible in the darkfield micrograph in Fig. $1 \mathrm{~d}$. The 16 fields of $10 \times 10 \mu \mathrm{m}^{2}$ are separated by additional, single cross-shaped markers written by He FIB. Each of the upper 12 fields contains 361 spot irradiations in a square grid of $19 \times 19$. The spot irradiations define the positions of the intended defect locations and are arranged with a grid spacing of either 250 or $500 \mathrm{~nm}$ with two repetitions to cover different areas on the EG (see annotations in Fig. 1d).

Based on molecular dynamics simulations the sputtering rate of $C$ atoms from graphene upon He ion irradiation with an energy of $30 \mathrm{keV}$ is expected to be very low ${ }^{23}$. However, the defect formation in 2D materials is not only determined by the ion mass and its energy, but is also affected by the interactions with the substrate, e.g. by back-scattered ions or atoms sputtered from the substrate. Hence, despite the low sputtering rate, by using enough He ions the FIB could remove more than only one $C$ atom per intended location of the IID. Therefore, the same square grids were written not only with varying distances, but also with varying ion numbers $(10,000,20,000$, or 40,000$)$ per intended location of the IID, to study the influence of the amount of He ions on the defect formation and the consequent growth of $\mathrm{h}-\mathrm{BN}$. The two
$10 \times 10 \mu \mathrm{m}^{2}$ fields in the lower right of the pattern remained blank without IIDs, serving as a reference for an unstructured sample area within the pattern. In the two squares to the lower left more complex shapes for the IIDs were drawn with the He FIB (not shown here).

Figure 1e compares the intensities of the $D\left(\sim 1375 \mathrm{~cm}^{-1}\right)$ and $G$ $\left(\sim 1600 \mathrm{~cm}^{-1}\right)$ Raman peaks of graphene in the different areas, with the mean spectra for the denser pattern with varying number of ions used per IID shown in Fig. If (the mean spectra of the pattern with 500 nm spacing is shown in Supplementary Fig. 2). For higher ion numbers a small increase of the D/G peak intensity ratio can be observed, indicating a slightly higher defect density ${ }^{26}$. Since the number of IID locations is constant in each pattern, this indicates the formation of more than one defect per intended defect location for higher amounts of $\mathrm{He}$ ions. For the $\mathrm{G}$ and $2 \mathrm{D}$ peak a shift to higher wavenumbers can be observed for the structured samples, which we attribute to charge transfer from adsorbates at the IIDs to the graphene after exposure to ambient conditions ${ }^{27}$. Mapping the $D$ peak intensity, we can distinguish the structured area of $19 \times 19$ defects in a dense pattern (with 40,000 ions per IID) from the surrounding pristine EG (inset of Fig. 1f).

\section{Selective-area van der Waals epitaxy of $\mathbf{h}$-BN on graphene}

Following the IID preparation, the substrates were introduced to the MBE system for the SAvdWE of h-BN islands (see Method section). Figure $2 a$ presents an atomic force microscopy (AFM) image of an area of $19 \times 19$ IIDs, each written with 20,000 ions, in a distance of $250 \mathrm{~nm}$ (areas with $500 \mathrm{~nm}$ distance can be seen in Supplementary Fig. 2). Areas with a rougher surface can be 

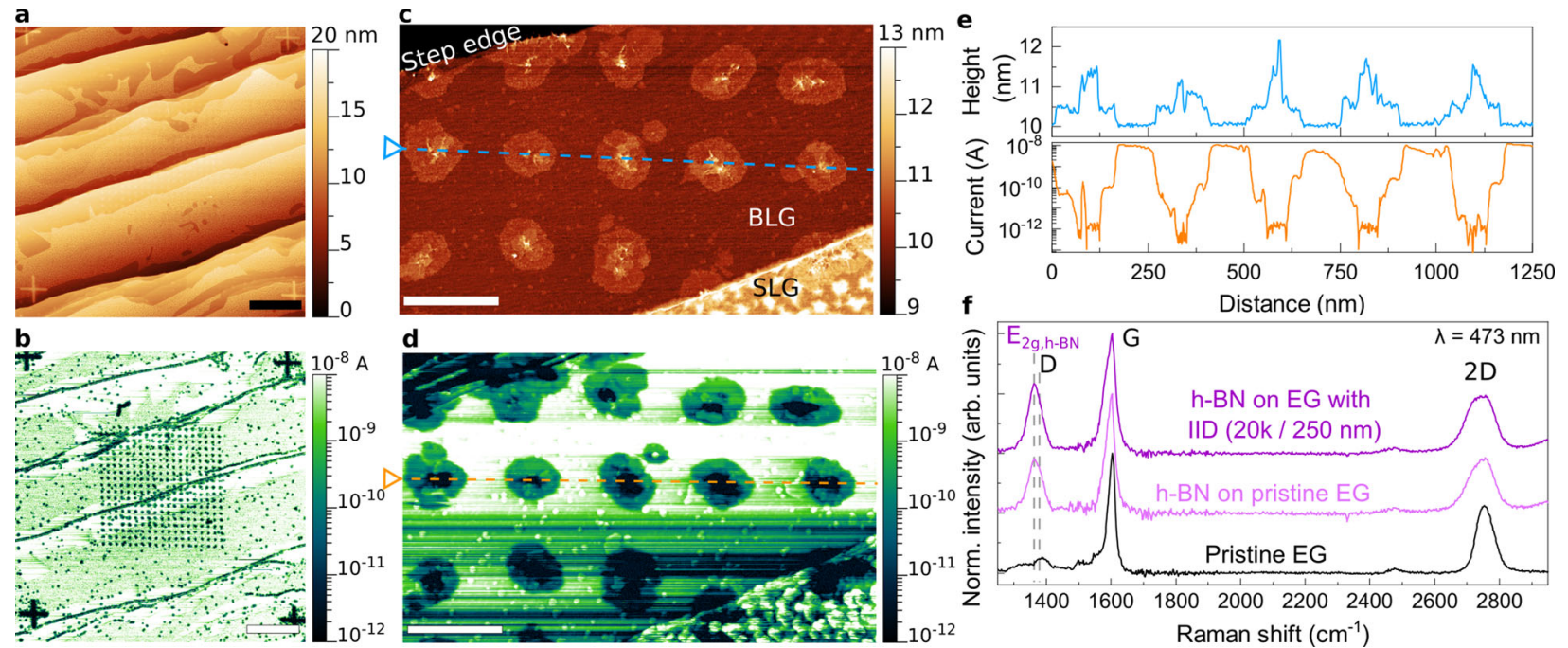

Fig. 2 h-BN islands grown via SAvdWE for $\mathbf{3 0 0}$ min. a AFM image of an area with $19 \times 19$ defects written with 20,000 ions per IID in a distance of $250 \mathrm{~nm}$ to each other between the cross-shaped markers. b Corresponding cAFM image with a bias of $100 \mathrm{mV}$ applied to graphene showing the position of the insulating h-BN islands. c Detailed AFM image of h-BN islands forming close to a step edge on BLG. d Corresponding CAFM image with a sample bias of $200 \mathrm{mV}$. e Height profile (upper graph) and corresponding current (lower graph) from the blue and orange dotted lines in (c) and (d), respectively. $\mathbf{f}$ Comparison of Raman spectra from pristine graphene with spectra from h-BN islands grown on non-structured areas as well as h-BN islands grown via SAvdWE. The spectra were normalized with regard to the G peak and are vertical shifted for clarity. Scale bars, $2 \mu \mathrm{m}$ in (a) and (b), $250 \mathrm{~nm}$ in (c) and (d).

distinguished from smoother areas close to the step edges, which we attribute to overgrown SLG and BLG, respectively, as shown in previous publications ${ }^{12,14}$. In EG, SLG is known to have a different chemical reactivity than $B L G$, due to an atomic corrugation of the $\mathrm{C}$-rich and partially $\mathrm{sp}^{3}$-bonded interface layer to $\mathrm{SiC}$, which is screened by an additional graphene layer in BLG, leading to the different growth behavior ${ }^{28,29}$. As the focus of the current study lies on the SAvdWE of h-BN islands at IIDs, we will concentrate in the discussion on the growth of h-BN at IIDs on BLG, where the influence of the interface layer, which is specific for EG on SiC, is negligible. The corresponding conductive AFM (CAFM) image of the same area (Fig. 2b) shows a clear contrast between graphene and $\mathrm{h}-\mathrm{BN}$ islands, which appear as insulating areas around the intended locations for the IIDs within the markers. Besides the nucleation at the IIDs, insulating h-BN can be also seen nucleating at the step edge and scarcely at natural defects, similar to what was observed in the previous studies ${ }^{14}$. In the detailed AFM image shown in Fig. 2c, a mean diameter of $150 \mathrm{~nm}$ of the h-BN islands can be observed on the BLG areas. Their average height of $0.34 \mathrm{~nm}$ (see also profile along the blue dotted line in Fig. 2e) corresponds well to previously reported values for h-BN islands grown on graphene and graphite ${ }^{11,14}$. The distance of $\sim 250 \mathrm{~nm}$ between the islands and their square arrangement precisely follow the FIB pattern. For single-crystalline h-BN islands either a triangular or hexagonal shape would have been expected ${ }^{30}$, but the islands here show a non-regular shape. Additional h-BN layers can be observed close to the center of the h-BN islands, together with $\sim 2 \mathrm{~nm}$ high nanoparticles (NPs), similar to the formation reported from naturally occurring defects in $\mathrm{EG}^{14}$. Fig. $2 \mathrm{~d}$ shows the corresponding CAFM image of the same area, where a clear contrast is observed not only between graphene and h-BN islands, but also within the islands, where the central area with the additional layers and NPs shows no tunneling current at a bias of $100 \mathrm{mV}$.

The Raman spectra of these samples shown in Fig. $2 f$ reveal a substantially increased intensity at $\sim 1360 \mathrm{~cm}^{-1}$, where the $E_{2 g}$ peak of h-BN is expected ${ }^{31}$. Similar to previous studies a shift of the $G$ and $2 D$ peak can also be observed, which can be attributed to a change in strain and doping of graphene ${ }^{12}$. In aligned h-BN/ graphene heterostructures fabricated via mechanical exfoliation a strained-induced broadening of the 2D peak (as we observe it here) was also reported ${ }^{32}$. Comparing the mean spectra of the patterned areas with non-patterned areas, a slightly higher intensity of the h-BN related Raman peak can be observed, which we attribute to the denser nucleation of $h-B N$.

\section{Controlling selective nucleation and orientation}

In Fig. 3a, b we compare the CAFM images of the areas structured with 40,000 and 10,000 ions per IID (corresponding AFM images are shown in Supplementary Fig. 3). For the higher amount of $\mathrm{He}$ ions in every location of an IID on the terraces a h-BN island can be found, while for fewer ions only in $86 \%$ of the IIDs a h-BN island nucleated (with most islands missing on BLG, see also Supplementary Fig. 3). The h-BN islands nucleating at IIDs written with 40,000 ions per IID have a diameter of $\sim 165 \mathrm{~nm}$ and a nonhexagonal shape (see Fig. 3c). Furthermore, the islands contain additional layers and a cluster of NPs in the center, indicating a similar formation as in the area studied before (see Fig. 2).

The morphology of the h-BN forming at IIDs written with 10,000 ions is shown in Fig. 3d. Several mono-layer thick h-BN islands on BLG present a hexagonal shape, with well-defined edges and without wrinkles. Their mean diameter amounts to $\sim 135 \mathrm{~nm}$ and they have a common alignment among each other (indicated by green dashed arrows). Furthermore, considering the well-known epitaxial relationship between EG and SiC (EG[1010] // $\mathrm{SiC}[1120])$ and the orientation of the surface step edges (see Fig. $1 \mathrm{~d})$, the results suggest that the edges of the $\mathrm{h}-\mathrm{BN}$ islands parallel to the green arrows follow the [1120] crystalline direction in EG. Unlike the non-hexagonal islands (see cAFM image in Fig. 3e), these oriented islands exhibit a Moiré pattern in their conductivity (Fig. 3f). The detailed CAFM image of an exemplary island (Fig. $3 \mathrm{~g}$ ) and the profile along the orange dotted line (Fig. $3 \mathrm{~h}$ ) reveal a periodicity of the Moiré pattern of $\sim 14 \mathrm{~nm}$. This value is expected for a relative rotation angle of $0^{\circ}$ between graphene and $h-B N$, and indicates epitaxially aligned $\mathrm{h}-\mathrm{BN}$ on graphene, with EG[1010]//hBN[1010], which is considered thermally stable ${ }^{33,34}$. A similar periodicity can be observed in all hexagonal-shaped islands. Hence, single-crystalline and epitaxially aligned h-BN islands can be grown via SAvdWE by defect engineering in the EG substrate 


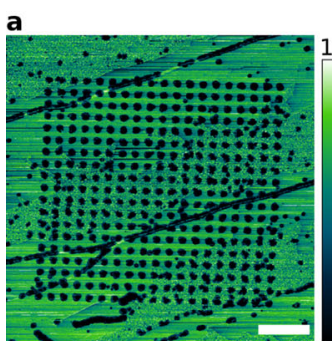

b

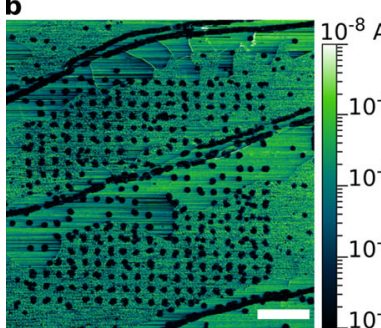

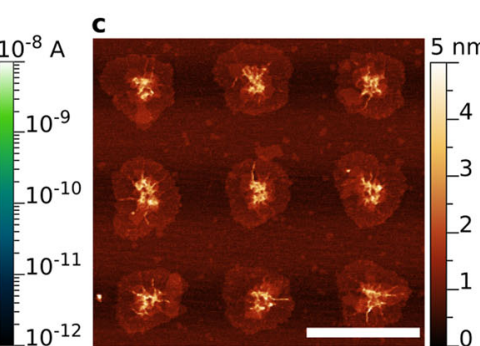

d

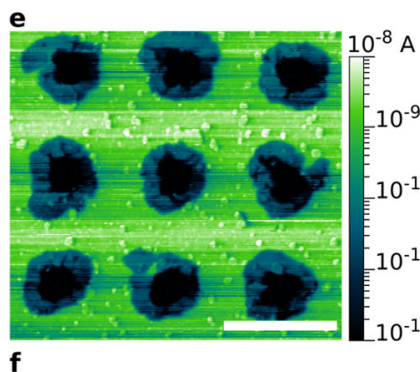

f
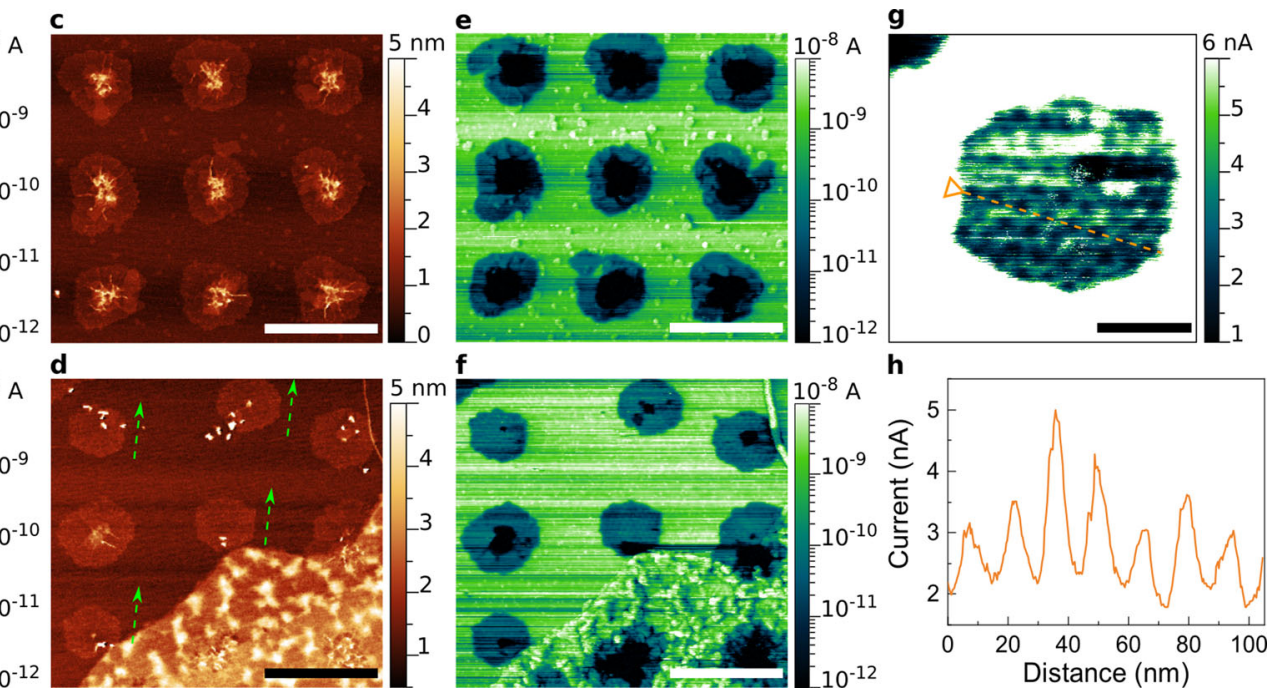

Fig. 3 SAvdWE of h-BN at IIDs written with varying number of He ions. CAFM images of areas with $19 \times 19$ overgrown IIDs in a distance of $250 \mathrm{~nm}$ to each other, written with (a) 40,000 and (b) $10,000 \mathrm{He}$ ions per IID. A bias of $100 \mathrm{mV}$ was applied to graphene during the measurements. c-f Detailed AFM images of h-BN islands forming close to a step edge on BLG patterned with (c) 40,000 and (d) 10,000 He ions per IID with corresponding CAFM images at a bias of $200 \mathrm{mV}$ in (e) and (f), respectively. The green dashed arrows in (d) act as a guide to they eye depicting the common orientation among the hexagonal-shaped $\mathrm{h}-\mathrm{BN}$ islands. $\mathbf{g}$ cAFM image of a single h-BN island with linear current scale. $\mathbf{h}$ Profile along orange dotted line in (g) demonstrates a Moiré pattern of $\sim 14 \mathrm{~nm}$ in the conductivity, evidencing an epitaxial alignment between the island and the EG. Scale bars, $2 \mu \mathrm{m}$ in (a) and (b), $250 \mathrm{~nm}$ in (c-f), $50 \mathrm{~nm}$ in (g).

using a He FIB. It is assumed, that this approach is not only limited to $\mathrm{h}-\mathrm{BN}$ on EG, but should be also applicable to other 2D materials, such as transition-metal dichalcogenides, which have been shown to preferentially nucleate at defects in $2 \mathrm{D}$ substrates as well ${ }^{15,35}$.

Furthermore, unlike in the non-hexagonal-shaped islands, the centers of the epitaxially aligned islands do not always coincide with the location of the IIDs. This could indicate that the defects written with $10,000 \mathrm{He}$ ions per IID may heal out or move during heating of the substrate to the growth temperature ${ }^{36}$. In contrast, we expect that using 20,000 or $40,000 \mathrm{He}$ ions per defect location resulted in the removal of multiple $C$ atoms per individual IID and therefore in the formation of laterally extended defects (e.g. nanoholes), which are more stable upon thermal treatment and allow for differently oriented $\mathrm{h}-\mathrm{BN}$ domains to nucleate at the same IID, resulting in the non-hexagonal-shaped $h-B N$ islands. Further studies will elucidate the nature of the IIDs and develop protocols for their stabilization.

The NPs on the well-aligned islands shown in Fig. 3d also got partially removed during the CAFM measurements (see Supplementary Fig. 4), indicating their relatively weak bonding to the h$\mathrm{BN}$ as compared to the clustered NPs observed in the islands nucleating at IIDs written with 20,000 and 40,000 He ions. This demonstrates that the deterministic placement of defects in the 2D substrate does not only affect the growth of the first 2D layer, but also the formation of additional layers.

\section{Lateral growth of h-BN layer from IIDs}

The vertical and lateral formation of the h-BN islands from the IIDs via SAvdWE was studied further by using an increased deposition rate of $B$ and longer growth time to form a coalesced $h-B N$ layer between the IIDs. The AFM image in Fig. 4a depicts an overgrown surface area in BLG, which was patterned with 20,000 ions per defect location, in a distance of $250 \mathrm{~nm}$ to each other. Inside the patterned area a completely coalesced h-BN mono-layer formed due to the higher nucleation yield at the IIDs, while outside of the pattern BLG remains still largely uncovered (see Supplementary Fig. 5). The chemical bonds in this sample were further studied via X-ray photoemission spectroscopy (see Supplementary Fig. 6) with values for the B1s and N1s peaks in agreement with values for $h-$ $\mathrm{BN}$ on $\mathrm{EG}^{14}$.

Apart from $\sim 8 \mathrm{~nm}$ high NPs some additional h-BN layers with diameters of up to $100 \mathrm{~nm}$ formed close to some IIDs, which have a relatively lower tunneling current, as shown in the CAFM image in Fig. 4b (some NPs got removed during the CAFM scan). Furthermore, between some differently oriented domains we observe lower tunneling currents at the grain boundaries, where the morphology shows a network of wrinkles. This suggests the formation of overlapping $h$-BN layers upon the coalescence of the different domains in these areas, similar to observations in h-BN grown via catalytic chemical vapor deposition on metal foils ${ }^{37}$. In other areas we also observe the formation of atomically smooth grain boundaries (see Fig. 4c), where differently oriented grains merged seamlessly. No increased tunneling current could be detected from either of these grain boundaries, which is a key aspect if such h-BN layers are to be employed as tunneling barriers.

The h-BN grain on the right-hand side of the CAFM image in Fig. $4 \mathrm{c}$ presents a Moiré pattern with a periodicity of $14 \mathrm{~nm}$, indicating the thermally stable relative rotation angle of $0^{\circ}$ between graphene and h-BN (as also observed in Fig. 3g). A Moiré periodicity of $3 \mathrm{~nm}$, as we observed it in the grain to the left-hand side of Fig. $4 \mathrm{c}$, indicates a relative rotation angle of $\sim 5^{\circ}$, which is thermally unstable ${ }^{34}$. We attribute the appearance of such unstable orientations in our sample to thermal expansion during cool down in combination with fixation of the h-BN domains at the IIDs.

In comparison to standard growth, another advantage of SAvdWE is the potential of controlling the grain boundary location by IID pattern design. Furthermore, neither the overlapping nor the seamless grain boundaries induced preferential formation of additional h-BN layers. Instead, additional layers can be found in the vicinity of the IIDs. This demonstrates that the artificially created defects can act as preferential nucleation points for the formation of additional h-BN layers on top of the first coalesced one. Thereby it appears plausible, that also other 2D materials could be connected to the IIDs upon completion of the first 2D 

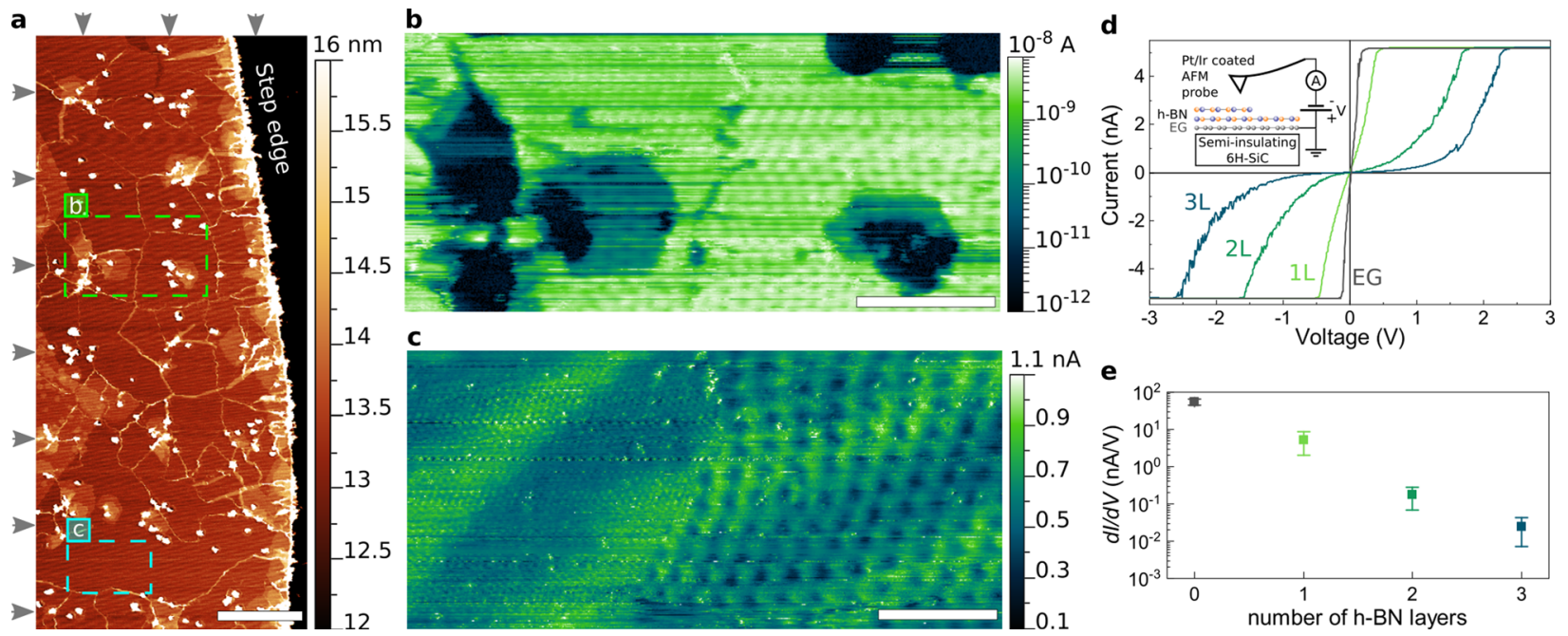

Fig. 4 Coalesced h-BN layers between IIDs in EG. a AFM images of an area close to a step edge with IIDs in a distance of $250 \mathrm{~nm}$ written with $20,000 \mathrm{He}$ ions per IID, overgrown with h-BN for $480 \mathrm{~min}$. Rows and columns of the IIDs with $250 \mathrm{~nm}$ spacing are marked by gray arrows. b CAFM image of the green framed region in (a), where multiple h-BN layers can be observed close to IIDs. c cAFM image of an atomically smooth area in between 4 IIDs as marked by the cyan colored frame in (a), demonstrating a grain boundary between two differently oriented domains, as evidenced by different periods of the Moiré patterns ( 3 and $14 \mathrm{~nm}$ ). A bias of $100 \mathrm{mV}$ was applied to graphene in (b) and (c). d Vertical transport measurements of $\mathrm{h}$-BN tunnel barriers, with representative $I-V$ curves of mono- (1L), bi-(2L) and tri-layer h-BN (3L) (inset: measurement configuration). e Log. scale tunnel conductance extracted from the linear regime of the $I-V$ curves. The error bars represent one standard deviation. Scale bars, $250 \mathrm{~nm}$ in (a), $100 \mathrm{~nm}$ in (b), $50 \mathrm{~nm}$ in (c).

layer, allowing more complex 2D heterostructures to form via SAvdWE. For the future device integration of such heterostructures, the active region could then be designed such, that it only incorporates the area in between the IIDs, where the heterostructures grow smoothly.

Figure $4 d$ shows representative $I-V$ curves from different locations on the samples with varying numbers of h-BN layers (the measurement configuration is depicted in the inset). The tunnel conductance shown in Fig. 4e was extracted from the linear regime of these $I-V$ curves and the observed exponential decay with increasing layer number is expected for direct tunneling of electrons. A similar tunneling behavior was observed for h-BN flakes, exfoliated from high-quality h-BN bulk crystals, showing that the electronic quality of the h-BN grown via SAvdWE can compete with heterostructures fabricated via mechanical transfer 38,39

In conclusion, we demonstrated a mask-free selective-area epitaxy of two-dimensional h-BN on graphene using a deterministic placement of defects via He FIB. The SAvdWE allowed an unprecedented control over the formation of epitaxially aligned and single-crystalline 2D heterostructures via a bottom-up approach. Additionally to the nucleation location, the lateral formation of $h-B N$ on EG was controlled via the growth parameter during the growth process, resulting in a 2D h-BN layer with tunneling characteristics identical to exfoliated material. Therefore, the SAvdWE contributes to precise and scalable fabrication of 2D heterostructures by establishing a reliable processing route for the site-selective, epitaxial bottom-up manufacturing with the potential for a reproducible fabrication of nano-devices based on 2D materials.

\section{METHODS}

\section{Substrate preparation}

The markers for the alignment of the FIB pattern were prepared on the $1 \times$ $1 \mathrm{~cm}^{2}$ large $6 \mathrm{H}-\mathrm{SiC}$ substrates with a miscut of $\sim 0.2^{\circ}$ using photolithography, followed by reactive ion etching using $\mathrm{SF}_{6}$. After chemically cleaning, the $\mathrm{SiC}(0001)$ was etched for $15 \mathrm{~min}$ at $14500^{\circ} \mathrm{C}$ in forming gas ( 5 at $\% \mathrm{H}_{2}$ in $\mathrm{Ar}$ ) using a flux of $500 \mathrm{sccm}$ at a pressure of $900 \mathrm{mbar}$. In a second step EG formed on the etched SiC via sublimation of Si for $30 \mathrm{~min}$ at $1650^{\circ} \mathrm{C}$ in $\mathrm{Ar}$ at a pressure of $900 \mathrm{mbar}$. Further information on the etching and sublimation process can be found elsewhere ${ }^{40,41}$. For the thermal coupling during the growth of $\mathrm{h}-\mathrm{BN}$ via MBE the backside of the $\mathrm{SiC}$ substrate was coated with $1 \mu \mathrm{m}$ of $\mathrm{Ti}$ via electron beam evaporation.

\section{He FIB patterning}

The samples were loaded to the FIB system one day in advance to the actual patterning to allow for desorption of adhering species during pumping over night. The background pressure during patterning was around $1 \times 10^{-7}$ mbar. After adjusting column and beam all stage moves and patterning tasks were carried out without imaging. Hence, unintended ion beam irradiation of the patterned regions could be avoided. The pattern was designed as described in the discussion of Fig. 1, with varying dwell times and distance between IIDs. Further information on the patterning and beam control can be found elsewhere ${ }^{42}$. The IIDs were prepared using a $30 \mathrm{keV} \mathrm{He}$ ion beam at an ion current of $1.8 \mathrm{pA}$. With the settings for the FIB used in this study, the dwell times were varied between 4,2 , and $1 \mathrm{~ms}$ corresponding to approximately 40,000, 20,000 and 10,000 ions per intended defect location, respectively. The appropriate ion number for creating defects which initiate nucleation was determined on test samples, where the ion dose was varied over several orders of magnitude covering the range from one ion per IID up to 130,000 ions. A typical test pattern for this purpose contained 6 individual IIDs as well as IIDs written along 6 lines, each $1 \mu \mathrm{m}$ long, to ensure sufficient statistics (see Supplementary Fig. 7).

\section{PAMBE of $h-B N$}

After loading the patterned EG samples into the MBE system, they were thermally cleaned in a preparation chamber for $30 \mathrm{~min}$ in UHV $\left(1 \times 10^{-9}\right.$ mbar) at $400^{\circ} \mathrm{C}$. Consecutively, the h-BN islands (coalesced h-BN layers) were grown for $300 \mathrm{~min}(480 \mathrm{~min})$ at a substrate temperature of $850^{\circ} \mathrm{C}$ using a high temperature effusion cell operated at $\sim 1850^{\circ} \mathrm{C}\left(1900^{\circ} \mathrm{C}\right)$ as a $\mathrm{B}$ source, whereas $\mathrm{N}$ was supplied using an Addon RFX450 RF plasma source operated at $350 \mathrm{~W}$ and a $\mathrm{N}_{2}$ flux of $0.2 \mathrm{sccm}$. The temperature was monitored using an optical pyrometer, and the heating and cooling rates were $\sim 20^{\circ} \mathrm{C} / \mathrm{min}$. Further information on the growth behavior of $\mathrm{h}-\mathrm{BN}$ on EG at similar conditions can be found elsewhere ${ }^{12,14}$.

\section{Micro-Raman spectroscopy}

Raman measurements were conducted in back-scattering configuration using an excitation wavelength of $473 \mathrm{~nm}$, with the laser focused on the 
sample using a $\times 100$ objective with a NA of 0.9 , resulting in a spot size of $\sim 1 \mu \mathrm{m}$. The reflected light was analyzed using a grating with $600 \mathrm{gr} . \mathrm{mm}^{-1}$ and a liquid $\mathrm{N}_{2}$ cooled Si CCD camera. Raman spectra were recorded for each pattern in a $5 \times 5 \mu \mathrm{m}^{2}$ map containing 36 measurement spots (each with $2 \times 60 \mathrm{~s}$ integration time) on the patterned sample with and without h-BN.

\section{AFM and CAFM}

The morphology of the samples was monitored using AFM in intermittent contact mode (tip diameter, $2 \mathrm{~nm}$; spring constant, $0.4 \mathrm{~N} / \mathrm{m}$ ) with the cantilever deflection used as feedback ${ }^{43}$. AFM scans were aligned with regard to the marker in the FIB pattern (e.g., see Fig. 1e and Supplementary Fig. 1). The CAFM measurements were conducted in contact mode at ambient conditions using $\mathrm{Pt} / \mathrm{lr}$ coated tips (tip diameter, $25 \mathrm{~nm}$; spring constant, $0.4 \mathrm{~N} / \mathrm{m}$ ). A bias between 100 and $200 \mathrm{mV}$ was applied to the EG on the semi-insulating $\mathrm{SiC}$ as described in the text, and the tunneling current between graphene and AFM tip was measured at a constant force of $\sim 10 \mathrm{nN}$. I- $V$ curves were recorded in $\mathrm{N}_{2}$ atmosphere at 144 locations in each of the areas shown in Fig. $4 a$, b with five repetitions per location.

\section{X-ray photoemission spectroscopy}

XPS measurements presented in Supplementary Fig. 6 were carried out with a Scienta Omicron Argus CU electron analyzer using a monochromated Al Ka X-ray source (Al $\mathrm{K}_{\mathrm{a}}: 1486.6 \mathrm{eV}$ ), with a spot size of a few millimeters in diameter.

\section{DATA AVAILABILITY}

The data supporting the findings of this work are available from the corresponding authors upon reasonable request.

Received: 10 December 2020; Accepted: 15 July 2021; Published online: 02 August 2021

\section{REFERENCES}

1. Novoselov, K. S., Mishchenko, A., Carvalho, A. \& Castro Neto, A. H. 2D materials and van der Waals heterostructures. Science 353, aac9439-aac9439 (2016).

2. Liu, C. et al. Two-dimensional materials for next-generation computing technologies. Nat. Nanotechnol. 15, 545-557 (2020).

3. Yankowitz, M., Ma, Q., Jarillo-Herrero, P. \& LeRoy, B. J. van der Waals heterostructures combining graphene and hexagonal boron nitride. Nat. Rev. Phys. 1, 112-125 (2019).

4. Lyu, J., Pei, J., Guo, Y., Gong, J. \& Li, H. A new opportunity for 2D van der Waals heterostructures: making steep-slope transistors. Adv. Mater. 32, 1906000 (2020).

5. Koma, A. Fabrication of ultrathin heterostructures with van der Waals epitaxy. J. Vac. Sci. Technol. B: Microelectron. Nanom. Struct. 3, 724 (1985).

6. Meng, J., Wang, D., Cheng, L., Gao, M. \& Zhang, X. Recent progress in synthesis, properties, and applications of hexagonal boron nitride-based heterostructures. Nanotechnology 30, 074003 (2019).

7. Mayorov, A. S. et al. Micrometer-scale ballistic transport in encapsulated graphene at room temperature. Nano Lett. 11, 2396-2399 (2011).

8. Lopes, J. M. J. Synthesis of hexagonal boron nitride: from bulk crystals to atomically thin films. Prog. Cryst. Growth Charact. Mater. 67, 100522 (2021).

9. Liu, Z. et al. Direct growth of graphene/hexagonal boron nitride stacked layers. Nano Lett. 11, 2032-2037 (2011).

10. Wang, M. et al. A platform for large-scale graphene electronics-CVD growth of single-layer graphene on CVD-grown hexagonal boron nitride. Adv. Mater. 25, 2746-2752 (2013).

11. Cho, Y. et al. Hexagonal boron nitride tunnel barriers grown on graphite by high temperature molecular beam epitaxy. Sci. Rep. 6, 34474 (2016).

12. Heilmann, M. et al. Influence of proximity to supporting substrate on van der waals epitaxy of atomically thin graphene/hexagonal boron nitride heterostructures. ACS Appl. Mater. Interfaces 12, 8897-8907 (2020).

13. Lin, Y.-C. et al. Direct synthesis of van der Waals solids. ACS Nano 8, 3715-3723 (2014).

14. Heilmann, M., Bashouti, M., Riechert, H. \& Lopes, J. M. J. Defect mediated van der Waals epitaxy of hexagonal boron nitride on graphene. 2D Mater. 5, 025004 (2018).
15. Zhang, X. et al. Defect-controlled nucleation and orientation of $\mathrm{WSe}_{2}$ on hBN: a route to single-crystal epitaxial monolayers. ACS Nano 13, 3341-3352 (2019).

16. Briggs, N. et al. A roadmap for electronic grade 2D materials. 2D Mater. 6, 022001 (2019).

17. $\mathrm{Li}, \mathrm{H}$. et al. Controlled synthesis of topological insulator nanoplate arrays on mica. J. Am. Chem. Soc. 134, 6132-6135 (2012).

18. Lin, M. et al. Controlled growth of atomically thin In 2 Se3 flakes by van der Waals epitaxy. J. Am. Chem. Soc. 135, 13274-13277 (2013).

19. Zhou, Y. et al. Epitaxy and photoresponse of two-dimensional gase crystals on flexible transparent mica sheets. ACS Nano 8, 1485-1490 (2014).

20. Munshi, A. M. et al. Selective area growth of AIGaN nanopyramid arrays on graphene by metal-organic vapor phase epitaxy. Appl. Phys. Lett. 113, 263102 (2018).

21. Lehtinen, O. et al. Effects of ion bombardment on a two-dimensional target: Atomistic simulations of graphene irradiation. Phys. Rev. B: Condens. Matter Mater. Phys. 81, 153401 (2010).

22. Ghorbani-Asl, M., Kretschmer, S., Spearot, D. E. \& Krasheninnikov, A. V. Twodimensional $\mathrm{MoS}_{2}$ under ion irradiation: From controlled defect production to electronic structure engineering. 2D Mater. 4, 025078 (2017).

23. Kretschmer, S. et al. Supported two-dimensional materials under ion irradiation: the substrate governs defect production. ACS Appl. Mater. Interfaces 10, 30827-30836 (2018).

24. Klein, J. et al. Site-selectively generated photon emitters in monolayer $\mathrm{MoS}_{2}$ via local helium ion irradiation. Nat. Commun. 10, 2755 (2019).

25. Yun, J. et al. Selective-area heteroepitaxial growth of h-BN micropatterns on graphene layers. 2D Mater. 5, 015021 (2018).

26. Cancado, L. G. et al. Quantifying defects in graphene via Raman spectroscopy at different excitation energies. Nano Lett. 11, 3190-3196 (2011).

27. Schedin, F. et al. Detection of individual gas molecules adsorbed on graphene. Nat. Mater. 6, 652-655 (2007).

28. Sclauzero, G. \& Pasquarello, A. Carbon rehybridization at the graphene/SiC(0001) interface: effect on stability and atomic-scale corrugation. Phys. Rev. B: Condens. Matter Mater. Phys. 85, 161405(R) (2012).

29. Bellucci, L., Cavallucci, T. \& Tozzini, V. From the buffer layer to graphene on silicon carbide: exploring morphologies by computer modeling. Front. Mater. 6, 1-6 (2019).

30. Stehle, Y. et al. Synthesis of hexagonal boron nitride monolayer: control of nucleation and crystal morphology. Chem. Mater. 27, 8041-8047 (2015).

31. Reich, S. et al. Resonant Raman scattering in cubic and hexagonal boron nitride. Phys. Rev. B 71, 205201 (2005).

32. Wang, Z. et al. Composite super-moiré lattices in double-aligned graphene heterostructures. Sci. Adv. 5, eaay8897 (2019).

33. Yankowitz, M. et al. Emergence of superlattice Dirac points in graphene on hexagonal boron nitride. Nat. Phys. 8, 382-386 (2012).

34. Wang, D. et al. Thermally induced graphene rotation on hexagonal boron nitride. Phys. Rev. Lett. 116, 126101 (2016).

35. Zhang, F. et al. Full orientation control of epitaxial MoS2 on hBN assisted by substrate defects. Phys. Rev. B 99, 155430 (2019).

36. Li, D. W. et al. Controlled defect creation and removal in graphene and MoS 2 monolayers. Nanoscale 9, 8997-9008 (2017).

37. Bayer, B. C. et al. Introducing overlapping grain boundaries in chemical vapor deposited hexagonal boron nitride monolayer films. ACS Nano 11, 4521-4527 (2017).

38. Lee, G. H. et al. Electron tunneling through atomically flat and ultrathin hexagonal boron nitride. Appl. Phys. Lett. 99, 243114 (2011).

39. Britnell, L. et al. Electron tunneling through ultrathin boron nitride crystalline barriers. Nano Lett. 12, 1707-1710 (2012).

40. Oliveira, M. H., Schumann, T., Ramsteiner, M., Lopes, J. M. J. \& Riechert, H. Influence of the silicon carbide surface morphology on the epitaxial graphene formation. Appl. Phys. Lett. 99, 111901 (2011).

41. Galves, L. A. et al. The effect of the SiC (0001) surface morphology on the growth of epitaxial mono-layer graphene nanoribbons. Carbon 115, 162-168 (2017).

42. Deinhart, V. et al. The patterning toolbox FIB-o-mat: exploiting the full potential of focused helium ions for nanofabrication. Beilstein J. Nanotechnol. 12, 304-318 (2021).

43. Kaemmar, S. B. Introduction to Bruker's ScanAsyst and PeakForce Tapping AFM Technology', Application Note 133, Rev. A0 (2011).

\section{ACKNOWLEDGEMENTS}

The authors would like to acknowledge fruitful discussions with Dr. Arkady Krasheninnikov on the irradiation-induced defect formation in 2D materials. M.H, A. T., and J.M.J.L. would also like to thank Werner Seidel for his help in the preparation of the patterned SiC substrates. Furthermore, they are grateful to Carsten Stemmler, Hans-Peter Schönherr, and Claudia Herrmann, for their dedicated maintenance of the 
PAMBE system and appreciate the critical reading of the manuscript by Dr. Alberto Hernández-Mínguez. V.D. and K.H. acknowledge financial support from DFG under grant no. HO 5461/3-1 and thank the European COST action CA19140 'FIT4NANO' (www.fit4nano.eu). The He ion beam patterning was performed in the Corelab Correlative Microscopy and Spectroscopy at Helmholtz-Zentrum Berlin.

\section{AUTHOR CONTRIBUTIONS}

M.H., K.H., and J.M.J.L. conceived and designed the experiments. M.H. and A.T. designed the mask for the photolithography used for marking the SiC substrates. A.T. supervised the photolithography processes. M.H. fabricated the EG samples on the marked SiC substrates. M.H., V.D., and K.H designed the mask for the FIB structuring. V.D. and K.H. performed the FIB structuring of the defects and characterized the samples using HIM and SEM. M.H. performed the MBE growth of $h-B N$ and characterized the samples using OM, AFM, TUNA, and Raman measurements. J.M.J.L. performed XPS measurements, supervised the project and advised on experiments and data analysis. M.H. wrote the manuscript. All authors discussed the results and commented on the manuscript.

\section{FUNDING}

Open Access funding enabled and organized by Projekt DEAL.

\section{COMPETING INTERESTS}

The authors declare no competing interests.

\section{ADDITIONAL INFORMATION}

Supplementary information The online version contains supplementary material available at https://doi.org/10.1038/s41699-021-00250-z.

Correspondence and requests for materials should be addressed to M.H. or J.M.J.L.

Reprints and permission information is available at http://www.nature.com/ reprints

Publisher's note Springer Nature remains neutral with regard to jurisdictional claims in published maps and institutional affiliations.

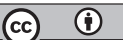

Open Access This article is licensed under a Creative Commons Attribution 4.0 International License, which permits use, sharing, adaptation, distribution and reproduction in any medium or format, as long as you give appropriate credit to the original author(s) and the source, provide a link to the Creative Commons license, and indicate if changes were made. The images or other third party material in this article are included in the article's Creative Commons license, unless indicated otherwise in a credit line to the material. If material is not included in the article's Creative Commons license and your intended use is not permitted by statutory regulation or exceeds the permitted use, you will need to obtain permission directly from the copyright holder. To view a copy of this license, visit http://creativecommons. org/licenses/by/4.0/.

(c) The Author(s) 2021 